\title{
IOT based Energy Management System for an Autonomous Hybrid Micro-Grid
}

\author{
S. Sivakumar, K. Siddappa Naidu, K. Karunanithi
}

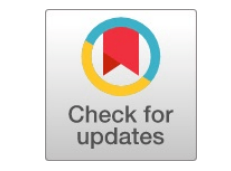

\begin{abstract}
Raising rate and require of power has led a lot of organizations to discover elegant ways for monitoring, controlling and reduction energy. To create an innovative idea to reduce the rate of energy consumption smart EMS (Energy Management System) is proposed in this paper. To develop IoT technologies and Big Data is used to improved hold energy utilization in commercial, housing and industrial sectors. An EMS is used to build smart homes is proposed for he developed cities. In this system, every residence tool is interfaced with a data attainment module that is an IoT object with an exclusive IP address ensuing in a huge mesh wireless network of devices. The data gaining SoC (System on Chip) module collects energy utilization data from every device of every stylish residence and send data to a centralized server for supplementary handing out and study. This information from all housing areas accumulates in the utility's server as Big Data. EMS consumes off-the-shelf BI (Business Intelligence) and Big Data surveys software packages which improves the energy usages also to assemble user order. While air conditioning gives to $60 \%$ of power use in American countries, HVAC (Ventilation, Air Conditioning and Heating) are in use as a research to approve the proposed system.
\end{abstract}

Keywords: Conditions, Cyber Security, Labview IOT (Gmail), GSM, Data Monitoring.

\section{INTRODUCTION}

In energy application, the most crucial and deliberated thought is considered for renewable sources of energy. The key adherence of the reusable source is termed as power management unit (PMU) and energy harvesting (EH) which gives the keen support to extract the source energy and make the extraction power to the synchronized module with respect to the underneath line voltage. The basic principle follows form kinetic energy and thermoelectric source [1]-[2]. With the modern world of technology, the sources of factor such as PMU and EH is linked to the cloud network with the help of internet of things (IOT) [1]-[3]. It is highly recommendable

Manuscript published on 30 August 2019.

*Correspondence Author(s)

S.Sivakumar, Research Scholar, Department of EEE, Vel Tech Rangarajan Dr.Sagunthala R\&D Institute of Science and Technology, Avadi, Chennai, Tamilnadu-600062, India. Email: siva.carthick@gmail.com.

K.Siddappa Naidu, Professor Emeritus, Department of ECE, Vel Tech Rangarajan Dr.Sagunthala R\&D Institute of Science and Technology, Avadi, Chennai, Tamilnadu-600062, India. Email: drksnaidu.blr@gmail.com.

K.Karunanithi, Associate Professor, Department of EEE, Vel Tech Rangarajan Dr.Sagunthala R\&D Institute of Science and Technology, Avadi, Chennai, Tamilnadu-600062, India. Email: k.karunanithiklu@gmail.com.

(C) The Authors. Published by Blue Eyes Intelligence Engineering and Sciences Publication (BEIESP). This is an open access article under the CC-BY-NC-ND license http://creativecommons.org/licenses/by-nc-nd/4.0/ by the society as it confines the high stability, low level of power dissipation at power on board, stabilized voltage supply and uniform power factor with conjunction of cloud network. Which resulting good power in tracing source and stabilizing the tracing power to the charging portion of $\mathrm{EH}$ bank [4]-[6]. Both these key module of reusable energy sources provides the synchronized charging and discharging among the sub module which express their state of art with higher efficiency harvesting and power managing principle. This property of IoT also involves for other solutions such as portable device, node sensor and wireless sensor network.

In this scenario, different thoughts sensing application such as physical sensor, chemical sensor, medical diagnosis etc have been emerged with regulated supply voltage [7]-[9] along with the connecting process module and minimum power consumption unit [10]-[11]. In this article, hybridization of both PMU with MPPT processing unit is discussed and the function decide the stable power supply to the EH system. The configuration of the line and load voltage is examined with high stability such that the entire block sustains with compatible mode. Further, PMU proficiency installed by 2D tuning circuit with MPPT controlling block whose dropout voltage is fed up by digital low dropout regulator. The internal converter at the input side of PMU supplies the internal regulated supply and it get the capable of stabilizing the voltage sources to the given input of EH.

\section{THEORY}

\section{A. Hybrid Grid System}

The isolated block diagram shown in Fig. 1

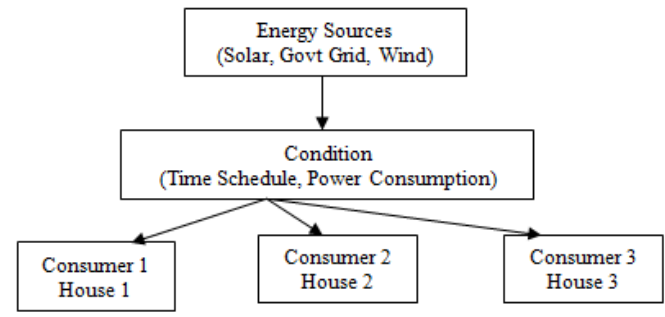

Fig. 1 System Overall Block Diagram

\section{B. Photo-Electric System}

In this system, the solar cell panel absorbs the energy and converts it corresponding DC source for the operation requirement of intermediate system. The main objective of the Converters acts for bride between DC source to the load voltage point and keeping the line under balance.

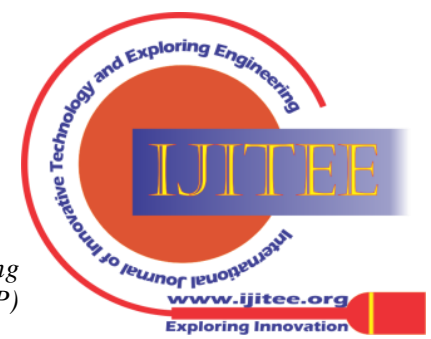




\section{IOT based Energy Management System for an Autonomous Hybrid Micro-Grid}

\section{Wind Kinetic System}

The pales while circulating the wind gives the electrical energy and it is obvious for acting as wind generator which acting as the winds kinetic energy and it tends to AC-DC for usable in the house.

\section{Battery Pack/EB}

Conversion of chemical energy into electrical energy that is stored is called as Battery.

\section{EXPERIMENTAL SETUP}

\section{A. IOT Based Energy Management System}

\section{- System Model}

Fig. 1 gives the fog computing protocol of IoT with three layers functional module. The assigned protocol splits the two nodes such that virtual top node (VTN) and virtual end node (VEN) which implies client and server respectively. After the assigning the node pointer and node addresses, tree formation between the VTN and VEN has been established. It is due to make the transparent transceiver properties. To ensure and stale the power supplying unit, the human machine interface between human and power grid is initialized [17]. The IEEE802.11 (WLAN) [18], IEEE 802.15 (WPAN) [19], and IEEE802.15.4 (LR-WPAN) [11]. In the design, the home gateway known as VEN which is exposed to the human power factor utilization and how much power consumption they have used and how amount of power cost is deduced in daily basis. All this information has been alerted by end node point of IOT structure. So that, the customers can get the aware that how amount of power we have consumed and how to balance the power consumption for remaining days until the tenure days of consumption bill settled.

In second layering module, fog node display in physical outfit fog execution gives wealth for services at the corner in the network. Fog nodes are positioned at the network corners and are close to the clients, late act is enhanced. In the planned design, the fog nodes contain CPU and storage capability that are used to execute the customer data also external requests. The fog nodes contain CPU and storage capability that are used to execute the customer data also external requests. To reduce stored data in the cloud servers, data aggregation protocols are employed at the network edge. Clients can control their thorough energy expenditure data securely using their local fog nodes. Besides, fog nodes reduce the search and access time with its locality attribute. From the TE view, the fog nodes serve as trade energy market server that serves energy services to the client. Energy trade markets supply the boundary between retailers and their customers. By calculating the previous and current consumption pricing point of grid, the retailing marketing pricing is fixed for the collection of customer side.

In third layer, the architecture is proposed for giving the stable assistance of data supplying and power consumption at the uniform distribution process. For example, the whole sale billing server has different system operator who enters the billing prices, purchase bills, returned protect etc., but the same cloud server maintains all data handling. Hence, it would be managed with uniform power utility at all network accessed system and for its IP address too.
- Conditions-Time Scheduling and Power

\section{Consumption}

Load complementary or load distribution is a plan to distribute workload in the middle of processors in a dispersed system. A few literatures compare both load balancing and load sharing. Load balancing is frequently distinct as a plan which attempts to promise that every computer in a system has equivalent load. On the other hand, Load sharing is more often than not referred to as a strategy which attempts to share loads in a distributed system without attempting to make equal its load. In the background of this learn, we shall use the term load sharing. A load sharing algorithm consists of 2 policies. A move strategy decides when a job be supposed to be transferred. This is more often than not determined based on the figure of jobs in the get in line waiting to be serviced. The site strategy decides to which host a job be supposed to be transferred. This is done also by choosing a crowd arbitrarily or by means of workload in order. The workload data is obtain also by inquisitive a subset of hosts or by meeting the data frequently. If the in order is composed occasionally, a best possible period has to be resolute. Collecting the information frequently will result in accurate.

\section{- Customer Model}

Fig.1 shows that customer energy usage depends upon the individuality and at the presence of neighbor surroundings. The variation of energy usage belongs to human intervention does not make any effect to supply the power through power grid for IoT system devices and its network adaptors. The gateway of installed IoT alerts daily about the human electricity usage, power consumption, unit calculation and calculation of bill accountable. The alerts from the network gateway make the aware of humans if he exceeded or not for power usage at that particular month. As a common, to secure the cloud activity of individual acting, fog computation is registered and needed so as it gives the more secure on Cloud data gate way. The stored data in private cloud is preserved by locally installed fog where the data is transferred with more secure to the common cloud server.

\section{- Cloud Computing}

The tough measures given still not possible within business constraints of an organization. In that state, a decision maker should execute a cost-benefit analysis to identify the trade-off over tough costs and network security. Also, a least amount cost hardening measure set only means that the core goal is safe, and some enduring damage may still remain in the network. Because these practical deals, network weakness management is not consider as a single-objective simplifying problem.

\section{- Cost Rendering}

At that time, the power supply intervention from the power grid is optimally transferred to the locally assisted fog network. The cost initiative will be delivered through the industry and the requested payment made via fog gateway where the transactional details will be preserved and more authenticated.

\section{- Prediction}

This architecture schedules the optimized plan and predicts the energy utilization factor under various PV generation [21]. 
The important and most effective prediction method is for time series analysis such that forecasting the historical happenings and composed for the future action. It could be done by the famous efficient algorithm known as normalized least mean square (NLMS) whose predictor key generates an estimation ${ }^{\wedge} \mathrm{F}(\mathrm{n}+\mathrm{k})$ of the value $\mathrm{F}(\mathrm{n}+\mathrm{k})$ that the process $\mathrm{F}$ will assume $\mathrm{k}$ steps ahead. The other special features of this prediction is that able to identify the repeated incident for the given the action of events.

\section{- Hardware Description}

In proposed system as presented in Mohammad Hossein Yaghmaee et. al [20], there are two sections one is transmitter section and next is receiver section. In transmitter section we can use four blocks which consists of RF transmitter, computer, max 232 and PIC16f877A is used. In receiver section $\mathrm{RF}$ receiver is used. The main part of the planned system is LPC 2148. Three sensors are used to for Power usage detection. The block design contains two section, transmitter section and receiver section. In transmitter part we can use four blocks which is computer, Max232, PIC16f877A, RF transmitter. We can give input to computer. In computer we can use software which is hyper terminal window. This data is transfer to MAX232. MAX 232 is largely accessed for communication systems in voltage is converted required to make TTL devices to be compatible with PC serial port and vice versa.PIC16f877A is used to convert data in four bits. $433 \mathrm{MHz} \mathrm{RF}$ transmitter is to transmit data. In receiver section receives data by using 433 $\mathrm{MHz}$ RF receiver. Received data is communicated to LPC 2148 and compared this data and then passed to next part.LPC2148 is the main part of the proposed system. Three sensors are identify the power usage.

\section{- Output Monitoring}

The output signal is processed by different node processor known as (RSCAD and RTDS). The enabling process of this node point has been done by triggering the input signal at each node point. The deliver pin can be described by DDAC and NI PXI which receives the controlling comment from the processor. The whole function is simulated by the Labview software and also experimentally verified. The information about the fault, repetition, final decision all are displayed by the front panel of node display point. The panel control point is enabled by the programmable logic control (PLC) and lead is in active stage followed by the control leads. The Labview program interfaces the monitoring unit with diagnosis patient. The collection of data are periodically repeated by the node processor and update the lookup table where the information about the client or patient or consumer purchase will be updated by data, time and month.

\section{RESULTS AND DISCUSSION}

The results and discussion section shows the collective performance of different power grid sector with different consumer information using common shared protocol known as IoT shared network clouds. Using Raspbian operating system, home based gateway has been developed and it has real time executed by raspberry pi3. The network adaptation with following hypertext transfer protocol (HTTP) to the home gateway point is successfully configured by the python 3.6.2 [12]. Further, the activation of HTTP could be enabled by java script with FX programing on support of Rasbery pi3 and the data collection to the IoT network is established configuring the point such as VTN,
VEN and Open AD [14]. Test bed and stacked protocol is configured for the communication link as shown in Fig.4. The gate way protocol is also configured in supporting IoT device through HTTP/CoAP method as given in Fig.5. The gate ways trigger pulse is uniformly spaced with bit slot duration of $15 \mathrm{~s}$. Hence, the synchronization between the IOT node devices with GET will be established well through HTTP protocol. Further, the response speed also improved between the sensor and process node at $200 \mathrm{~K}$. The input data is then executed for conveying the information to the delegates' node through gateway protocol followed by the fog and cloud node. The same communication transverse protocol is executed after every $15 \mathrm{sec}$ of gate trigger pulse. Both these way of communication establishment will be properly monitored by UDP datagram and HTTP protocol.

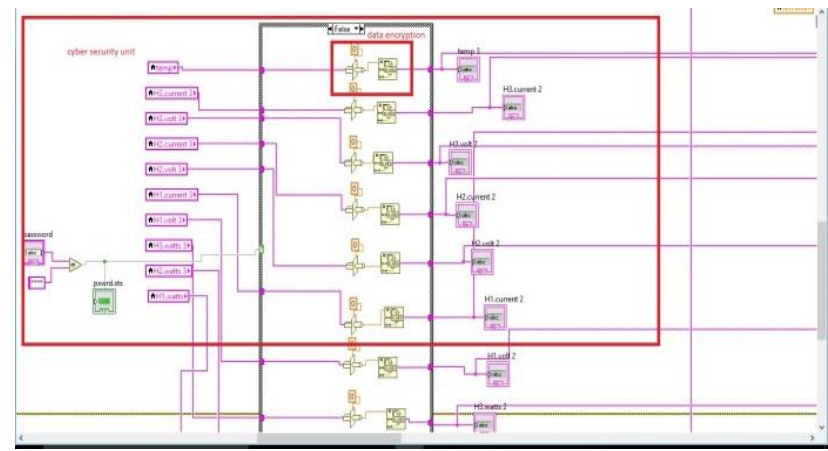

Fig. 2. Cyber Securities

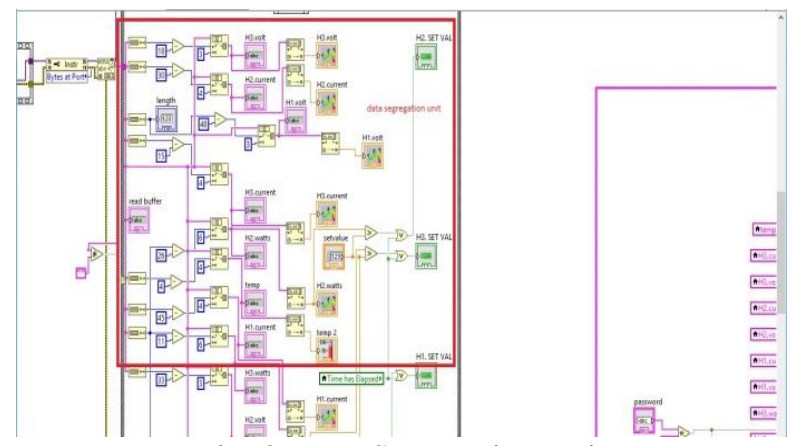

Fig. 3. Data Segregation Unit

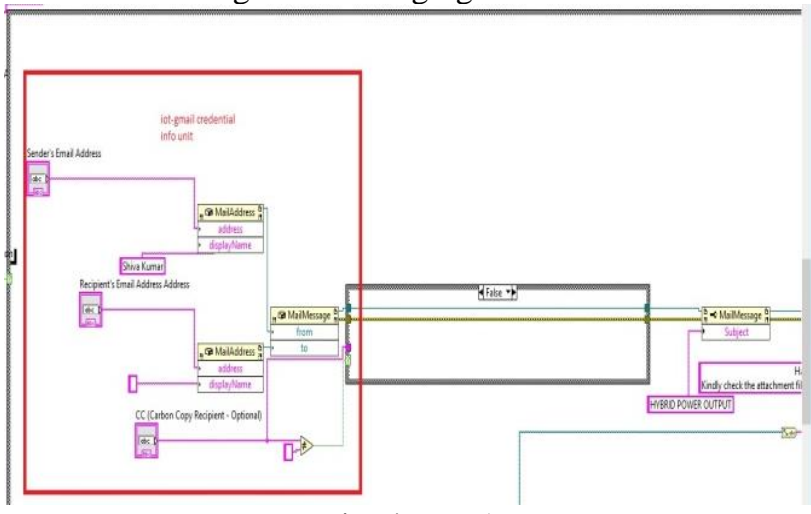

Fig. 4. IoT 1

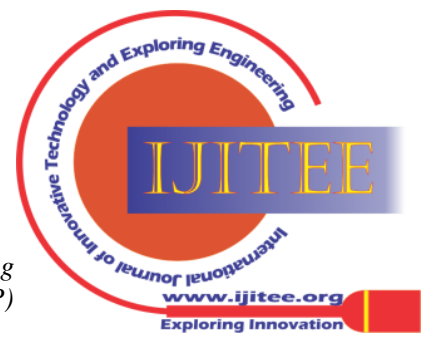




\section{IOT based Energy Management System for an Autonomous Hybrid Micro-Grid}

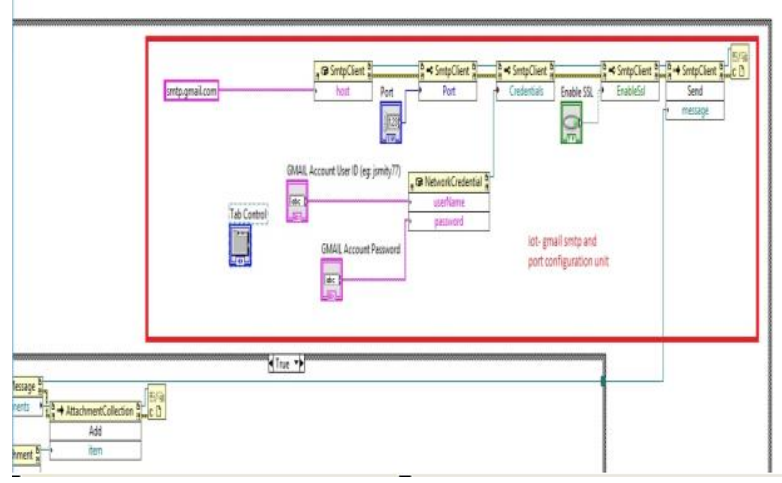

Fig. 5. IoT 2

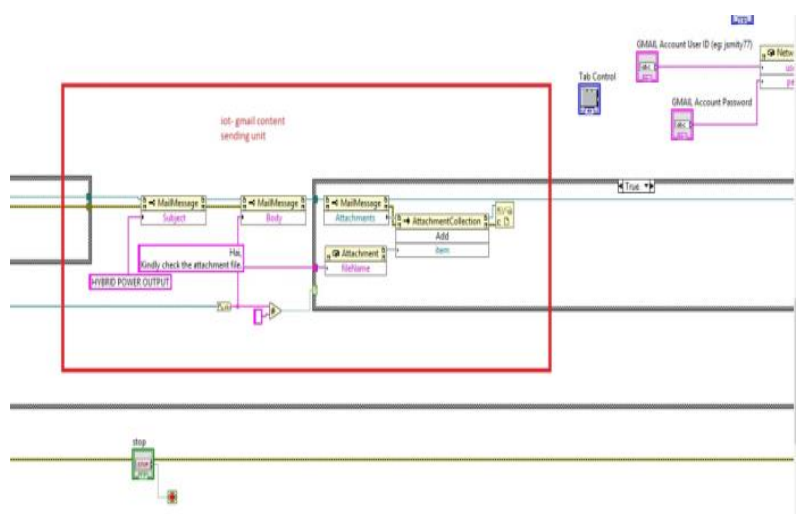

Fig. 6. IoT 3

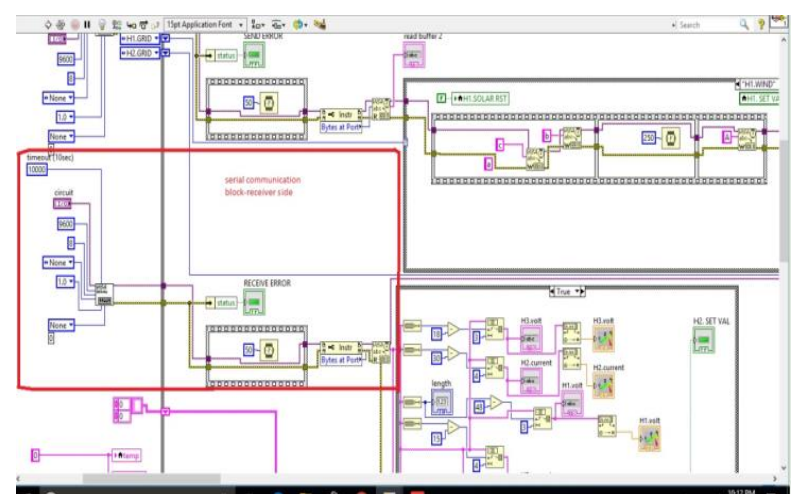

Fig. 7. Receiever Side

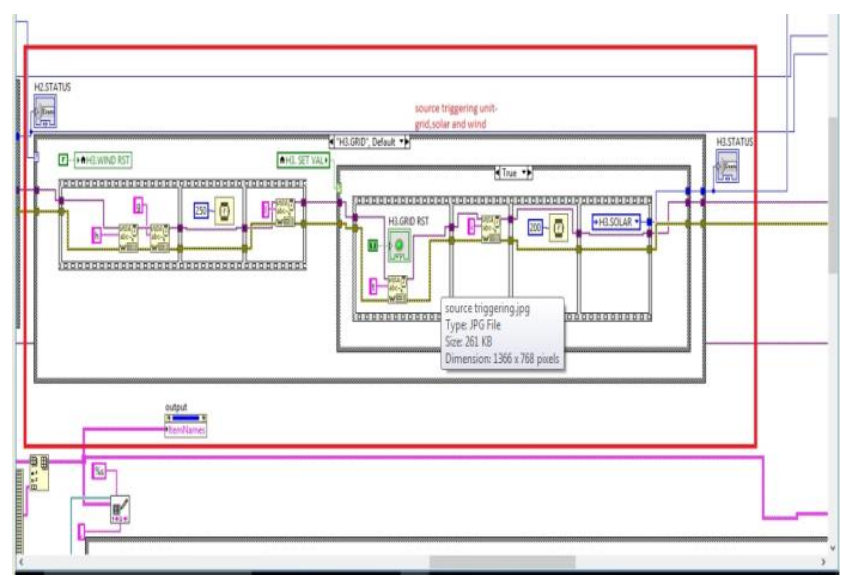

Fig. 8. Source Triggering

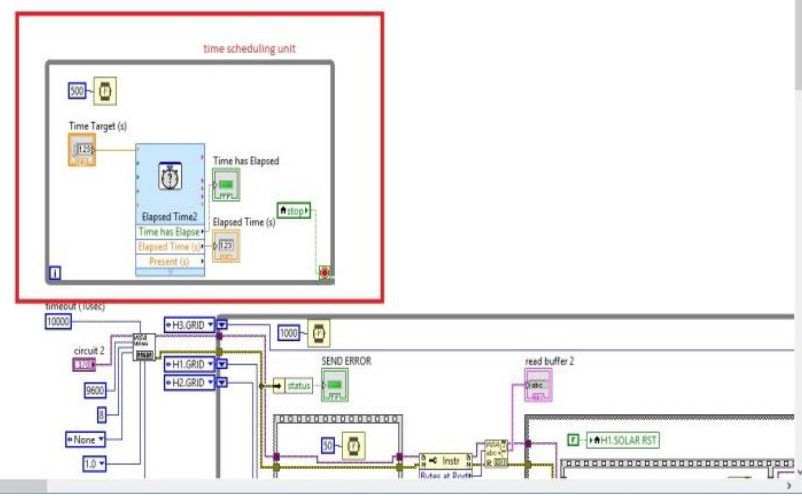

Fig. 9. Time Scheduling Unit

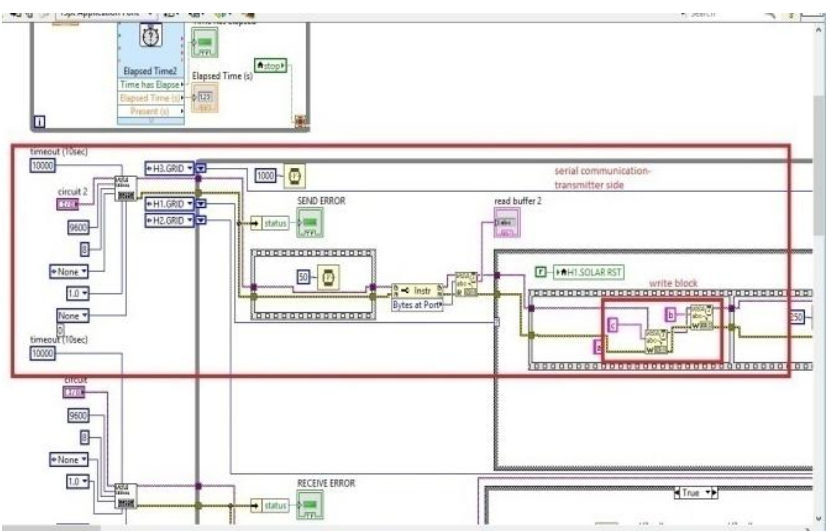

Fig. 10. Check New Min Delay.Ver.1

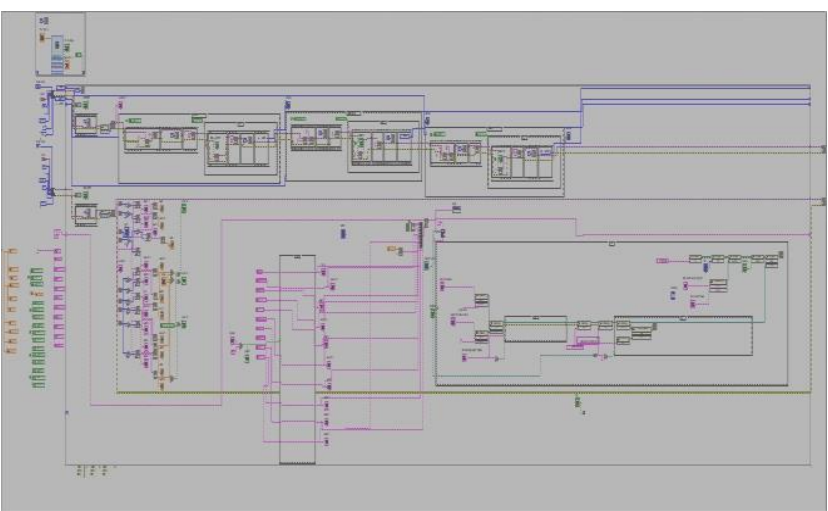

Fig. 11. Block Diagram

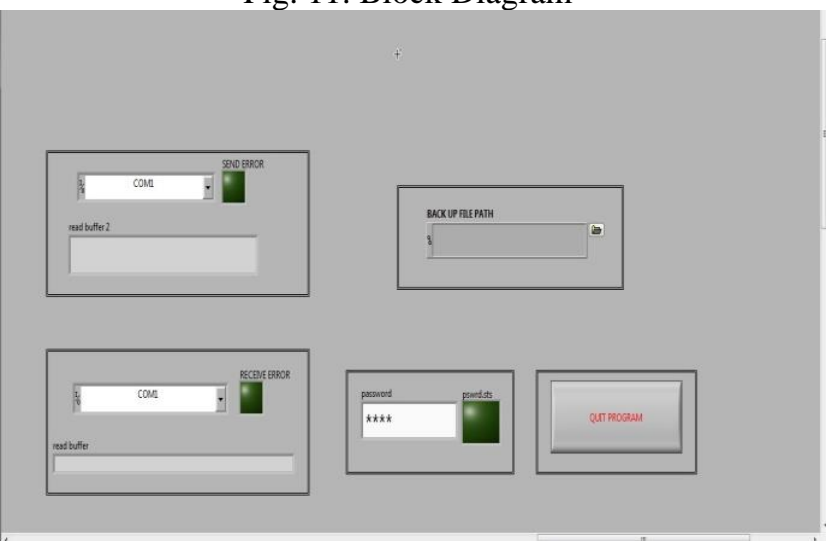

Fig. 12. Check New min delay.Ver.1.Front Panel 1

Published By:

Blue Eyes Intelligence Engineering

and Sciences Publication (BEIESP)

DOI: 10.35940/ijitee.I8171.0881019

Journal Website: www.ijitee.org

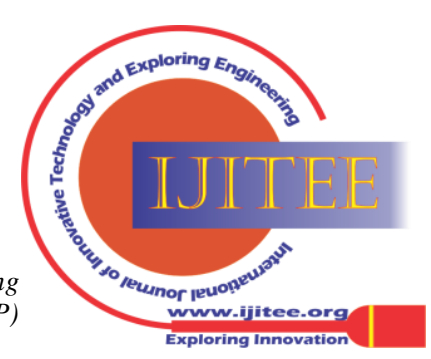




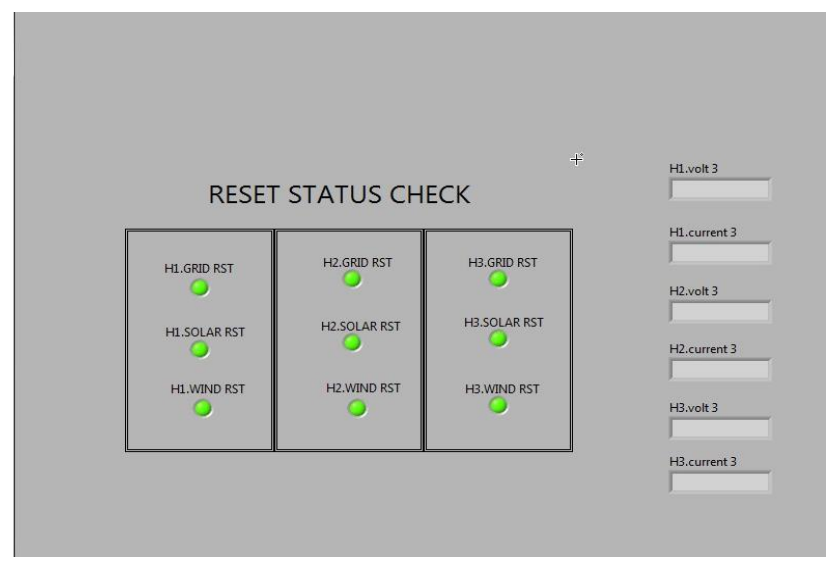

Fig. 13. . Check New min delay.Ver.1.Front Panel 2

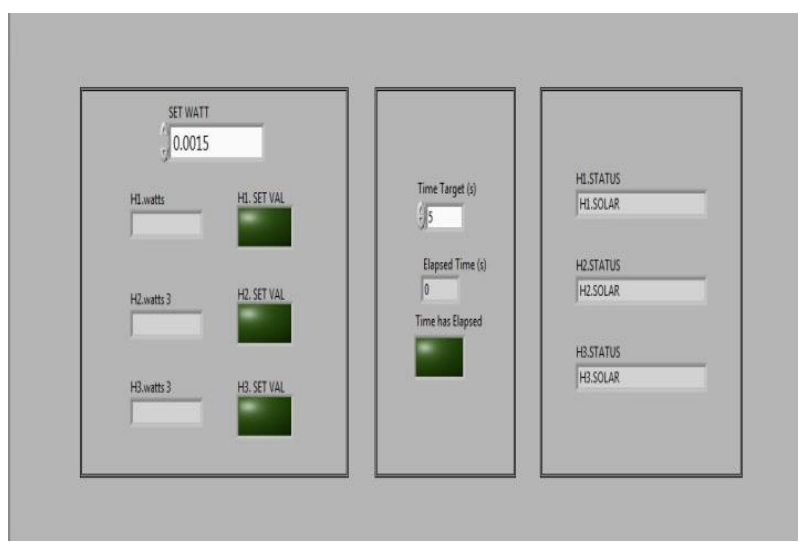

Fig. 14. Check New min delay.Ver.1.Front Panel 3

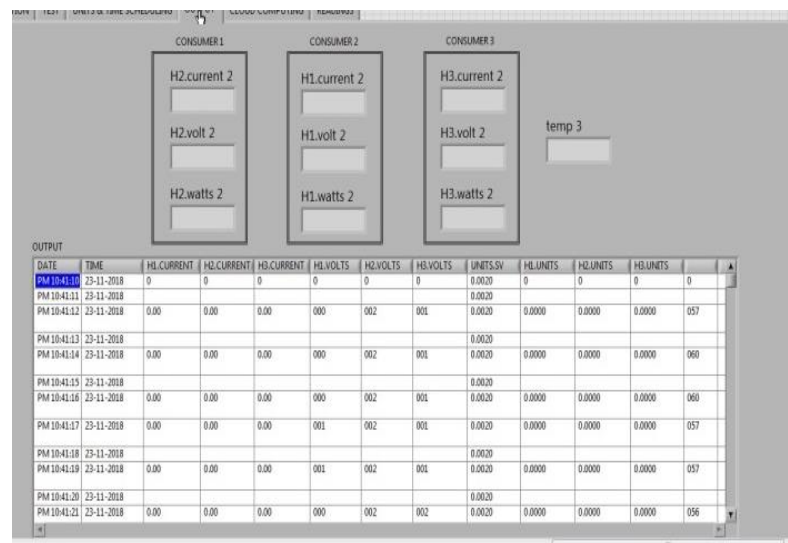

Fig. 15. Check New min delay.Ver.1.Front Panel 4

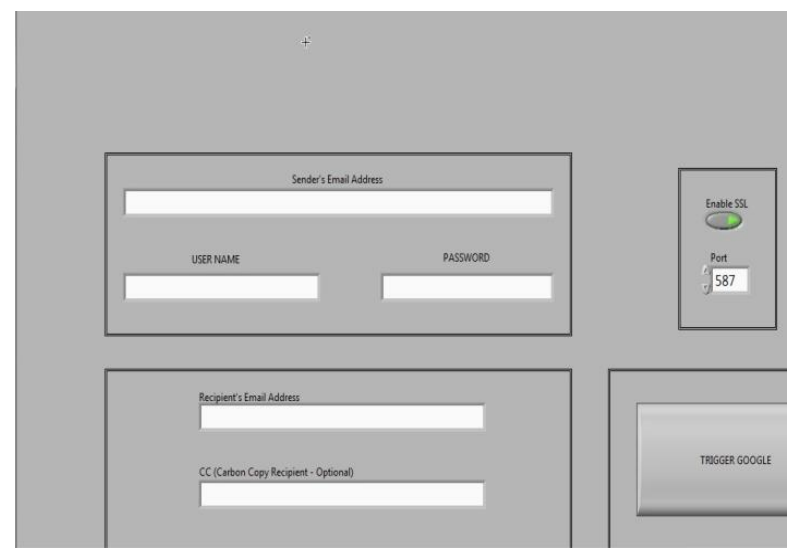

Fig. 16. Check New min delay.Ver.1.Front Panel 5

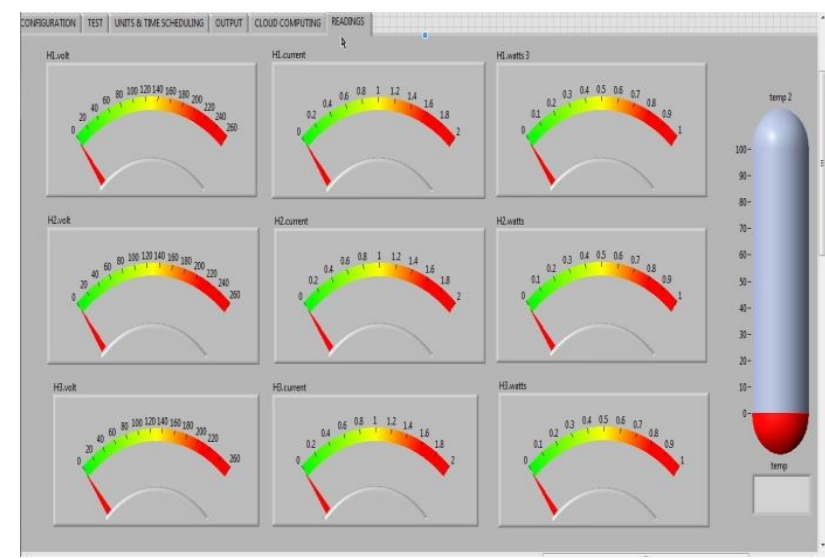

Fig. 17. Check New min delay.Ver.1.Front Panel 6

\section{CONCLUSION}

In this article, the power management unit based on the multitier communication system was investigated. The proposed architecture includes the fog station, cloud access, home gateway, power grid and harvesting system. The function of home gateway is fixed to collect the consumer data form the consumer to common cloud server through its gateway itself. The consumer information such as utilization factor, power consumption in daily basis and weekly basis, power rated by the appliances, power bill cost calculation were all conveyed to the home gate way using ADR protocol process. This primary alert was used to make the smart decision for consumer side to avoid their billing with more cost. In other aspects, TE supported DR program was studied and employed for going with optimized schedule of customer appliances.

\section{REFERENCES}

1. Y. Lee et al., "A modular $1 \mathrm{~mm} 3$ die-stacked sensing platform with low power I2C inter-die communication and multi-modal energy harvesting", IEEE J. Solid-State Circuits, vol. 48, no. 1, pp. 229-243, Jan. 2013.

2. Y. Zhang et al., "A battery less $19 \mu \mathrm{W}$ MICS/ISM-band energy harvesting body sensor node SoC for ExG applications," IEEE J. Solid-State Circuits, vol. 48, no. 1, pp. 199-213, Jan. 2013.

3. S. Bandyopadhyay and A. P. Chandrakasan, "Platform architecture for solar, thermal, and vibration energy combining with MPPT and single inductor," IEEE J. Solid-State Circuits, vol. 47, no. 9, pp 2199-2215,Sep. 2012

4. S. Bandyopadhyay, P. P. Mercier, A. C. Lysaght, K. M. Stankovic, and A. P. Chandrakasan, "A $1.1 \mathrm{nW}$ energy-harvesting system with $544 \mathrm{pW}$ quiescent power for next-generation implants," IEEE J. Solid-State Circuits, vol. 49, no. 12, pp. 2812-2824, Dec. 2014.

5. Y. K. Teh and P. K. T. Mok, "Design of transformer-based boost converter for high internal resistance energy harvesting sources with 21 $\mathrm{mV}$ self-startup voltage and 74\% power efficiency," IEEE J. Solid-State Circuits, vol. 49, no. 11, pp. 2694-2704, Nov. 2014.

6. W. Jung et al., "An ultra-low power fully integrated energy harvester based on self-oscillating switched-capacitor voltage doubler", IEEE Solid-State Circuits, vol. 49, no. 12, pp. 2800-2811, Dec. 2014.

7. Bosch BMP180 Digital Pressure Sensor, 2015, http://aebst.resource.bosch.com/media/products/dokumente/bmp180/B ST-BMP180-DS000-12 1.pdf.

8. CAT34TS00 1.8 V Digital Temperature Sensor, On Semiconductor 2014 ,

9. Data Sheet, SHTC1 Humidity and Temperature Sensor IC, ver. 4, Sensirion Aug. 2015,

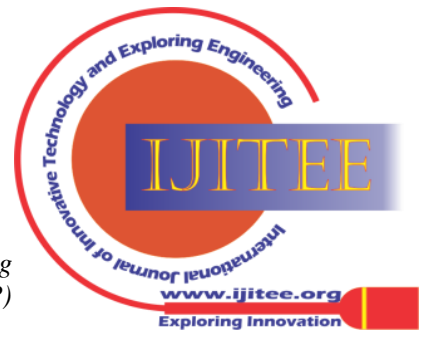




\section{IOT based Energy Management System for an Autonomous Hybrid Micro-Grid}

http://www.sensirion.com/fileadmin/user upload/customers/sensirion/ Dokumente/Humidity/Sensirion_Humidity_SHTC1_Datasheet_V3.pdf

10. "What the Internet of Things (IoT) Needs to Become Reality," Free scale Semiconductor/ARM, Inc. White Paper, 2012-2014.

11.R. Yu and T. Watteyne, "Reliable, low power wireless sensor networks for the internet of things: Making wireless sensors as accessible as webservers,"

http://cds.linear.com/docs/en/white-paper/wp003.pdf.

12. G. Palumbo and D. Pappalardo, "Charge pump circuits: An overview on design strategies and topologies," IEEE Circuits Syst. Mag., vol.10,no.1, pp. $31-45,2010$

13.E. J. Carlson, K. Strunz, and B. P. Otis, "A $20 \mathrm{mV}$ input boost converter with efficient digital control for thermoelectric energy harvesting," IEEEJ. Solid-State Circuits, vol. 45, no. 4, pp. 741-750, Apr. 2010.

14. P. S. Weng, H. Y. Tang, P. C. Ku, and L. H. Lu, "50 mV-input battery-less boost converter for thermal energy harvesting," IEEE J. Solid-State Circuits, vol. 48, no. 4, pp. 1031-1041, Apr. 2013.

15. Y.-C. Shih and B. P. Otis, "An inductor less DC-DC converter for energy harvesting with a $1.2 \mu \mathrm{W}$ bandgap-referenced output controller," IEEE Trans. Circuits Syst. II, Exp. Briefs, vol. 58, no. 12, pp. 832-836, Dec.2011.

16. J. Zarate Roldan, S. Carreon Bautista, A. Costilla Reyes and E. Sanchez Sinencio “ An Ultra-Low power management unit with -40dB switching noise suppression for a $3 \times 3$ thermoelectric generator array with $57 \%$ maximum end to end efficiency", Proc. IEEE custom integrated circuits conference, San Jose, California, US, 2014, pp. 1-4.

17. I. Doms, P. Merken, C. Van Hoof, and R. P. Mertens, "Capacitive power management circuit for micro power thermoelectric generators with a $1.4 \mu \mathrm{A}$ controller," IEEE J. Solid-State Circuits, vol. 44, no. 10,pp. 2824-2833, Oct. 2009

18. Y. H. Lee et al., "A low quiescent current asynchronous digital-LDO with PLL-modulated fast-DVS power management in $40 \mathrm{~nm} \mathrm{SoC} \mathrm{for}$ MIPS performance improvement," IEEE J. Solid-State Circuits, vol. 48 , no. 4,pp. 1018-1030, Apr. 2013.

19. S. Gangopadhyay, D. Somasekhar, J. W. Tschanzand and A. Raychowdhury, "A 32nm embedded fully-digital, phase-locked low drop out regulator for fine grained power management in digital circuits," IEEE J. Solid-State Circuits, vol. 49, no. 11, pp. 2684-2693, Nov. 2014.

20. Yaghmaee Moghaddam, M. H., \& Leon-Garcia, A. (2018). A Fog-Based Internet of Energy Architecture for Transactive Energy Management Systems. IEEE Internet of Things Journal, 5(2), 1055-1069. doi:10.1109/jiot.2018.2805899.

\section{AUTHORS PROFILE}

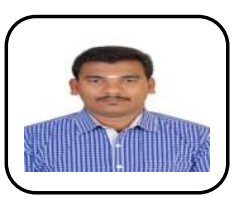

S.Sivakumar has completed his B.E. in Electrical and Electronics Engineering from V.R.S. College of Engineering and Technology, Arasur, Villupuram, Tamilnadu, India in 2007 and M.E. in Embedded System Technology from Veltech Engineering College, Avadi, Chennai, Tamilnadu, India under Anna University Affiliation, Chennai, Tamilnadu, India in 2009. At present, he is working as an Associate Professor in the Department of Electrical and Electronics Engineering at Vel Tech Rangarajan Dr.Sagunthala R\&D Institute of Science and Technology, Avadi, Chennai, Tamilnadu, India and doing his Ph.D. at the same Institution in the field of Renewable Energy with Cloud Computing. He has published many dissertations in International Conferences and Journals in his research field. His research area of interests are in Embedded System Networking, Cloud Computing, Internet of Things, Smart Grid and Renewable Energy Systems.

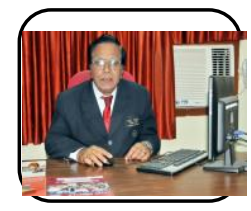

Prof. K.Siddappa Naidu completed his B.E. Electrical and Electronics Engineering from Sri Venkateswara University, Tirupati in 1973 and got Post Graduation from IISc, Bengaluru in 1976 and $\mathrm{Ph} . D$. from the same Institute in 1994. He has worked in various capacities in NGEF Transformers Research \& Development from 1979 to 2000 . He served as HoD-EEE, Dean-SoEC, Vice Principal and Principal in different Engineering Colleges and Universities from 2000 to 2014. At present time, he working as an Emeritus Professor in the Department of Electronics and Communication Engineering in Vel Tech Rangarajan Dr.Sagunthala R\&D Institute of Science and Technology, Avadi, Chennai-600062, Tamilnadu, India. He has published many dissertations in International and National Journals. His research area of interests are in Partial Discharge Measurements in HV Insulation and Apparatus, Online Monitoring of HV Power Apparatus, Sub Synchronous, Renewable Energy Systems and Power Quality.

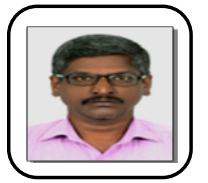

Dr. K.Karunanithi received the AMIE. Degree from the Institution of Engineers (India), Kolkata and M.E., Degree (with Distinction) from Anna University, Tirunelveli, India. He is awarded doctoral degree from Kalasalingam University, Krishnankoil, Tamilnadu, India. He is presently working as an Associate Professor in the Department of Electrical and Electronics Engineering at Vel Tech Rangarajan Dr.Sagunthala R\&D Institute of Science and Technology, Avadi, Chennai, Tamil Nadu, India. He has published many dissertations in International Conferences and Journals in his research field. His research area of interests are in Power System Planning and Power Systems.

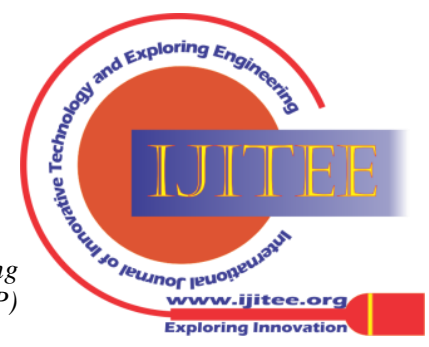

